\title{
In the pursuit of equity: COVID-19, data and Aboriginal and Torres Strait Islander people in Australia
}

\author{
Kalinda Griffiths ${ }^{\mathrm{a}, \mathrm{b}, \mathrm{c}, *}$, Ian Ring ${ }^{\mathrm{d}}$, Richard Madden ${ }^{\mathrm{e}}$ and Lisa Jackson Pulver ${ }^{\mathrm{e}, \mathrm{f}}$ \\ ${ }^{a}$ Centre for Big Data Research in Health, University of New South Wales, Sydney, Australia \\ ${ }^{\mathrm{b}}$ Wellbeing and Preventable Chronic Diseases Division, Menzies School of Health Research, Darwin, Australia \\ ${ }^{\mathrm{c}}$ Melbourne School of Population and Global Health, University of Melbourne, Melbourne, Australia \\ ${ }^{\mathrm{d}}$ Tropical Health \& Medicine, James Cook University, Cairns, Australia \\ ${ }^{\mathrm{e}}$ Faculty of Medicine and Health, University of Sydney, Sydney, Australia \\ ${ }^{\mathrm{f}}$ Office of the Deputy Vice-Chancellor (Indigenous Strategy and Services), University of Sydney, Sydney, Australia
}

\begin{abstract}
Since March 2020 in Australia, there has been decisive national, and state and territory policy as well as community led action involving Aboriginal and Torres Strait Islander people as information about COVID-19 arose. This has resulted in, what could only be framed as a success story in self-determination. However, there continues to be issues with the quality of data used for the surveillance and reporting of Aboriginal and Torres Strait Islander people during the pandemic. This article discusses some of the important events in pandemic planning regarding Aboriginal and Torres Strait Islander people and how this relates to surveillance and monitoring in the emerging and ongoing threat of COVID-19 within Aboriginal and Torres Strait Islander communities. The authors also identify some of the data considerations required in the future to monitor and address public health.
\end{abstract}

Keywords: COVID-19, surveillance, official statistics, indigenous peoples, Aboriginal and Torres Strait Islander people, policy, Australia

\section{Introduction}

Since the first case of Novel Coronavirus (COVID19 ) in Australia in late January 2020, there has been significant action by the Federal Government to coordinate existing state and territory ${ }^{1}$ public health systems and to support preparedness and emergency response measures that focus on Aboriginal and Torres Strait Islander people. ${ }^{2}$ Since March 2020, there has been decisive national, and state and territory policy as well as

\footnotetext{
*Corresponding author: Kalinda Griffiths, Menzies School of Health Research, PO Box 41096, Casuarina, Northern Territory, 0811, Australia. Tel.: +61 41884 7575; Fax: +61 88946 8464; E-mail: kalinda.griffiths@unsw.edu.au.

${ }^{1}$ Australian states include Western Australia, Queensland, New South Wales, South Australia, Victoria and Tasmania. There are also two mainland territories, specifically, the Australian Capital Territory and the Northern Territory.

${ }^{2}$ This article uses the terms Aboriginal and Torres Strait Islander people, Aboriginal people, First Nations Peoples and Indigenous
}

community led action involving Aboriginal and Torres Strait Islander people as information about COVID-19 arose. This has resulted in, what could only be framed as a success story for Australia, where Aboriginal and Torres Strait Islander people have had only 147 cases at the time of writing, with no deaths [1]. This has been largely due to the immediate and extensive efforts of the Aboriginal Community Controlled Organisations (ACCHOs) to support their members as well as their partnered leadership with the Australian Government to provide timely responses to COVID-19.

However, while there has been a comprehensive pandemic policy response across the nation, the efforts in collating and using data has provided clear insights to the existing gaps at different stages during the COVID-

people with recognition and respect that there are over 400 distinct Aboriginal and Torres Strait Islander Peoples (clan groups/languages) across Australia. 
Table 1

2016 Aboriginal and Torres Strait Islander Estimate Resident Population by state and territory and interface availability Aboriginal and Torres Strait Islander information on Government COVID-19 webpages (Dec 2020)

\begin{tabular}{lrrcc}
\hline & $\begin{array}{c}\text { Total } \\
\text { Australian } \\
\text { population }\end{array}$ & $\begin{array}{c}\text { Indigenous } \\
\text { population }\end{array}$ & $\begin{array}{c}\text { Proportion } \\
\text { of total } \\
\text { Indigenous } \\
\text { population }\end{array}$ & $\begin{array}{c}\text { Proportion } \\
\text { of total } \\
\text { population }\end{array}$ \\
\hline NSW & $7,732,858$ & 265,685 & 33.3 & 3.4 \\
Vic & $6,173,172$ & 57,767 & 7.2 & 0.9 \\
QLD & $4,845,152$ & 221,276 & 27.7 & 4.6 \\
SA & $1,712,843$ & 42,265 & 5.3 & 2.5 \\
WA & $2,555,978$ & 100,512 & 12.6 & 3.9 \\
Tas & 517,514 & 28,537 & 3.6 & 5.5 \\
NT & 245,678 & 74,546 & 9.3 & 30.3 \\
ACT & 403,104 & 7,513 & 0.9 & 1.9 \\
Australia & $\mathbf{2 4 , 1 8 6 , 2 9 9}$ & $\mathbf{7 9 8 , 3 6 5}$ & $\mathbf{n / a}$ & $\mathbf{3 . 3}$ \\
\hline
\end{tabular}

SOURCE: Australian Bureau of Statistics: Estimates of Aboriginal and Torres Strait Islander Australians, June 2016.

19 sequence of events. While there has been review on the success of Aboriginal and Torres Strait Islander leadership and National Aboriginal Community Controlled Organisations (NACCHO) efforts in the COVID19 preparedness response [2], to date there has been limited discussion on the health and social impact of COVID-19 on Aboriginal and Torres Strait Islander people in Australia. This raises questions about the facilitators and barriers to the surveillance and monitoring of Aboriginal and Torres Strait Islander people during a pandemic. This article discusses some of the important events in pandemic planning regarding Aboriginal and Torres Strait Islander people and how this relates to surveillance and monitoring in the emerging and ongoing threat of COVID-19 within Aboriginal and Torres Strait Islander communities. The authors also identify some of the data considerations required in the future to monitor and address public health.

\section{Aboriginal and Torres Strait Islander peoples}

At last census, the population estimate of Aboriginal and Torres Strait Islander people was 798,365, comprising 3.3 percent of the total Australian estimated population [3]. Table 1 shows the proportion of Aboriginal and Torres Strait Islander peoples of the total populations of the states and territories [3]. The Northern Territory (NT) has the highest proportion of Aboriginal and Torres Strait Islander people at 30.3 percent, and New South Wales (NSW) has the most Aboriginal and Torres Strait Islander people of any of the states and territories at 265,685. About $64 \%$ of Aboriginal and Torres Strait Islander people live outside of capital city areas [3].

\section{COVID-19 policy action}

In response to the COVID-19 pandemic, the Australian Government acted quickly. The Chief Medical Officer and the state and territory Chief Medical Officers developed and published the 'Australian Health Sector Emergency Response Plan for Novel Coronavirus (COVID-19)' (The Plan) in March 2020 [4]. This built on the existing 'Australian Health Management Plan for Pandemic Influenza' initially published in 2008 and updated in 2014 and again in 2019 [5]. The Plan has a strong focus on using existing systems and dynamic approaches to reduce the risk and impact of COVID19 on people and health care systems. There are several federal primary response plans covering a range of topics including technology and surveillance that have been developed and supported by the Commonwealth. The Australian Health Protection Principal Committee, which is the peak decision-making committee for disease control in Australia, signs off on these plans and coordinates the national response that is enacted by the states and territories. This has resulted in a total of 28,047 cases and 908 total deaths from COVID-19 since 22 January to 16 December 2020 . A relatively successful achievement compared to most other nations [6].

To support and guide the development of a 'National Aboriginal and Torres Strait Islander COVID-19 Management Plan', an Aboriginal and Torres Strait Islander Advisory Group on COVID-19 was convened in March 2020 [7]. By May 2020, this had resulted in an extensive range of measures supported by the Australian Government including:

- The establishment of General Practice Respiratory Clinics across Australia including within Aboriginal Community Controlled Organisations

- A rapid Point of Care Testing Program for COVID19 in rural and remote Aboriginal and Torres Strait Islander communities

- The development of COVID-19 infection control training programs specific for remote health services

- Facilitating Government support for the planning and preparedness activities in remote communities

- Advising the travel restrictions to remote communities

- A remit to improve data collection and Aboriginal and Torres Strait Islander identification in healthcare and pathology testing [8]

Importantly, the recognition of the subsequent impacts of pandemics upon individual and community mental health was acknowledged as priority for Abo- 


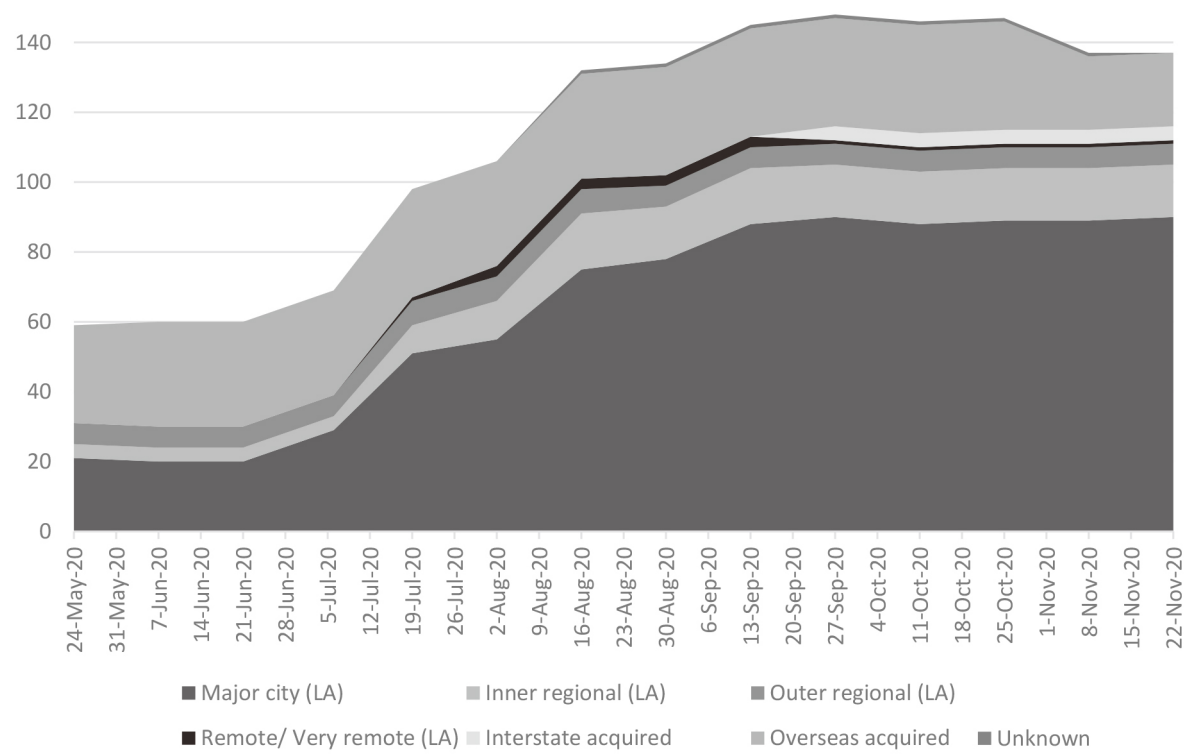

Fig. 1. Aboriginal and Torres Strait Islander COVID-19 cases by source of acquisition and remoteness classification. LA = Locally acquired SOURCE: Commonwealth Department of Health, Communicable Diseases Intelligence COVID-19 Epidemiology Reports (report dates shown in figure).

riginal and Torres Strait Islander people. This is due to the disproportionate impacts of pandemics upon Indigenous people globally as well as the inadequate and inequitable access to mental health care that Aboriginal and Torres Strait Islander people face, despite known ongoing mental health disadvantages [9]. At the time of writing the majority of Aboriginal and Torres Strait Islander COVID-19 cases were in major cities and only one was remote or very remote (Fig. 1) [1]. Despite this, there remains serious concerns for regional and remote Aboriginal and Torres Strait Islander communities due to the higher rates of other health issues and inadequate access to health care.

\section{Learning from $\mathrm{H} 1 \mathrm{~N} 1$}

Since March 2020, there has been decisive policy and community led action by Aboriginal and Torres Strait Islander people as information about the COVID19 pandemic arose. This was in part due to the existing risks and conditions that are likely to facilitate the spread of infectious diseases being higher than for nonIndigenous people. Specifically, this includes higher rates of chronic conditions as well as inadequate and inequitable access to health care services. Of particular interest to infectious respiratory disease are risk factors such as smoking and overcrowding, which is higher among Aboriginal and Torres Strait Islander people living remotely (Fig. 2).
It is also in part due to the impact that previous pandemics have had regarding Aboriginal and Torres Strait Islander people. While Australia had developed a national approach to the potential threat of a pandemic through the 'Australian Health Management Plan for Pandemic Influenza', there was limited input and ongoing surveillance relevant to Aboriginal and Torres Strait Islander people within the framework [5]. Within this plan, Aboriginal Community Organisations and Aboriginal General Practitioners were included as 'consultative fora' [5]. While consultation with peak bodies in Australia is critical to decision making and ground level actions, there was limited opportunity for multitiered input and support regarding governance for those working on the ground. The first wave of 2009 Influenza A (nH1N1) lasted about 18 weeks in Australia and resulted in an age-standardised Indigenous to nonIndigenous mortality rate ratio of 5.8 [10]. This is a contrasting story to the mortality ratio seen during the current COVID-19 response.

Aboriginal and Torres Strait Islander people experience ongoing disproportionate impacts due to infectious diseases. While there continues to be incremental annual reductions in the overall burden of disease impacting Indigenous people in Australia, infectious disease continues to have higher relative burden when compared to non-Indigenous Australians [11]. Aboriginal and Torres Strait Islander people are also at higher risk of infectious disease impact due to greater risk factors, 


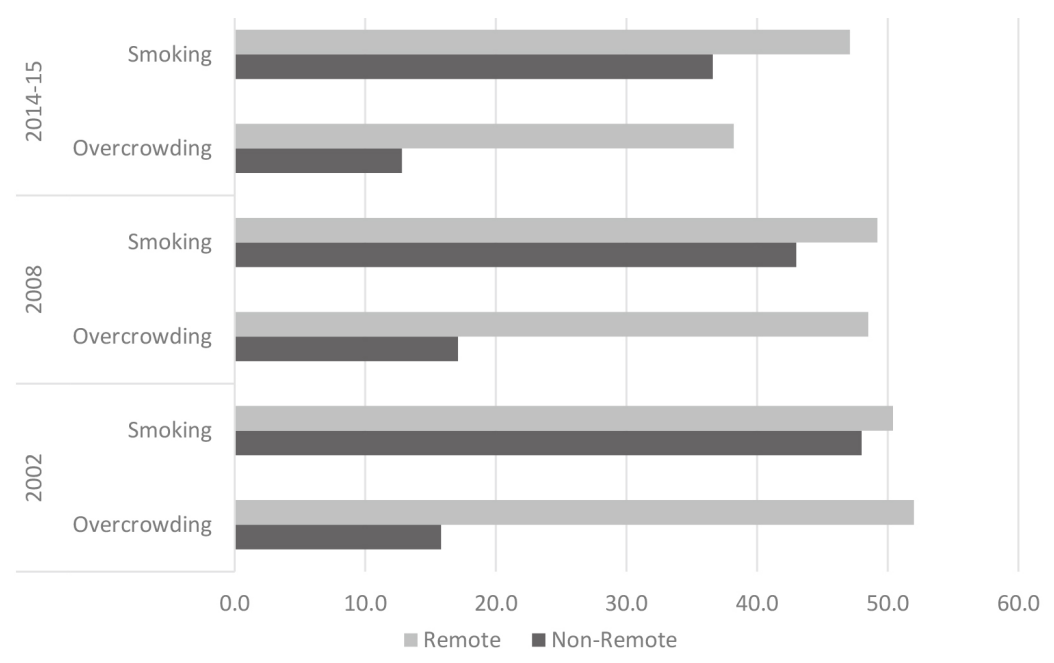

Fig. 2. Aboriginal and Torres Strait Islander overcrowding and daily smoking by remoteness and year. SOURCE: Australian Bureau of Statistics. 2016. 4714.0 National Aboriginal and Torres Strait Islander Social Survey, Australia, 2014-2015.

pre-existing and comorbid conditions and reduced access to services. Importantly, increased disease severity and fatality from COVID-19 is associated with greater age, obesity, diabetes and hypertension [12]. Further, during pandemics, attendance at primary health care services reduces due to concerns of spread [13]. Historically, Aboriginal and Torres Strait Islander people also experienced disproportionate hospital admissions and ICU admissions during the nH1N1 pandemic [14].

This reporting stressed the known issues of infectious disease risk to Aboriginal and Torres Strait Islander people. It also llustrated the requirement for setting up a public health system, particularly for surveillance and monitoring, that could work to better support Aboriginal and Torres Strait Islander people and communities. It identified the challenges in the quality of Indigenous status reporting, highlighting that at the time only four of the eight states and territories (Northern Territory, Queensland, South Australia, Western Australia) had adequate data to develop national Aboriginal and Torres Strait Islander estimates [10]. This also speaks to the well-known issues of the under identification and issues with the quality of Aboriginal and Torres Strait Islander data within surveillance and official reporting in Australia [15]. The reality of under-reporting, and excess morbidity and mortality will not be properly known for some time. Currently, the actual effect of the COVID19 pandemic on Australia's First Nations Peoples and communities is difficult to estimate. None the less, the role of the Aboriginal Community Controlled Sector cannot be under-estimated.

\section{Decision making and governance to support Aboriginal and Torres Strait Islander self-determination}

The critical mechanisms of the COVID-19 success story in Australia included the action to involve Aboriginal and Torres Strait Islander people in decisionmaking processes. This also involved higher-level governance processes to include and support Aboriginal and Torres Strait Islander people and communities. This is one of the first times in Australia's history where Commonwealth decision making created space for Indigenous autonomy within governance processes.

There have been ongoing prescribed limited rights of Aboriginal and Torres Strait Islander people from Australian governments since colonial settlement. The injustice that Aboriginal and Torres Strait Islander people have experienced due to government policies have resulted in continuing negative impacts upon cultural continuity and community integrity, as well as the health, social and emotional wellbeing [16]. This has furthered systemic discrimination against Aboriginal and Torres Strait Islander people within processes of self-determination, decision making and governance development.

The achievements of government agencies incorporating processes that enable self-determination for Aboriginal and Torres Strait Islander people within the COVID-19 context speaks for itself. It also reiterates the importance for Aboriginal and Torres Strait Islander people to have mechanisms for voice into governments and their decision-making processes for those discus- 
sions that impact Aboriginal and Torres Strait Islander people. There are a range of overarching mechanisms that can support voices to governments. This includes treaties, which is a formal agreement between governments and Aboriginal and Torres Strait Islander people to enable legal recognition and sovereignty as well as provide a basis for self-government [17]. Australia has never signed a treaty despite ongoing discussions and advocacy regarding the issue. ${ }^{3}$ Additionally, the Uluru Statement from the Heart ('Uluru Statement') sought to have a First Nations Voice enshrined in the Australian Constitution and requested to the establishment of a Makarrata Commission to supervise a process of agreement-making and truth telling between the Australian Government and Aboriginal and Torres Strait Islander people [18]. Requests which to date have not been accepted by government. Importantly, timely and accurate population level and small area estimates that can describe health issues and the needs of Aboriginal and Torres Strait Islander people are critical in these discussions. Furthermore, timely and accurate population estimates can better direct resources and facilitate health system developments. This in turn will enable and support Aboriginal and Torres Strait Islander sovereignty and self-determination.

\section{COVID-19 data and Aboriginal and Torres Strait Islander people ${ }^{4}$}

Australia has a well established communicable disease surveillance system, the National Notifiable Diseases Surveillance System (NNDSS) which was founded in 1990. The system operates at the national, state and local jurisdictional levels. The states and territories collect notifications of communicable diseases under their public health legislation. ${ }^{5}$ The health departments within each of the states and territories are also responsible for the public health action required. This

\footnotetext{
${ }^{3}$ Victoria, Western Australia, Northern Territory and Queensland all have commenced formal processes to develop Aboriginal and Torres Strait Islander treaties in their respective states and territories.

${ }^{4}$ These concepts were written and compiled into a global brief on Covid-19, Data and Indigenous peoples for the United Nations Statistical Division from the International Group for Indigenous Health Measurement Australian Caucus.

${ }^{5}$ There are a number of Australian state, territory and national public health laws. The Communicable Diseases Network Australia (CDNA) developed the 'CDNA National Guidelines' for public health units amidst the COVID-19 pandemic for Australia. These guidelines align with legislation and have been endorsed by the Australian Health Protection Principal Committee.
}

typically provides a basis for developing public health policy and for identifying and responding to communicable disease outbreaks of national significance. While this should be good news for population surveillance of COVID-19, there are well known challenges in accurate collection and reporting of Aboriginal and Torres Strait Islander data.

Data challenges were picked up in the National Aboriginal and Torres Strait Islander COVID-19 Management Plan where the improvement of data collection and Aboriginal and Torres Strait Islander identification in healthcare and pathology testing was prioritised as a primary remit [4]. These are national challenges that have always plagued population level data collections and particularly so since the implementation of the Standard Indigenous Question in 1996 [15].

The authors have noted a range of data matters in the surveillance and reporting of COVID-19 in Aboriginal and Torres Strait Islander data which includes, although is not limited to:

- Data coverage

- Data quality

- Provision of access to Aboriginal and Torres Strait Islander data.

This is not an extensive overview of these issues, but merely a snapshot of some of the focus points that are required in the efficient collection and reporting of data to inform decision making from and with Aboriginal and Torres Strait Islander people and communities during a pandemic.

\subsection{Data coverage}

While the NNDSS provides communicable disease surveillance, there is lacking publicly available infrastructure and systematic collection of data to inform public health policy and primary health care during a pandemic. Several points regarding data coverage have arisen as issues in the Aboriginal and Torres Strait Islander context and include:

- Health systems and services

- Workforce requirements

- Community/regional infrastructure

- Access to primary health care

- Education/Health promotion.

The ability for appropriate pandemic planning, management and responsiveness to occur is reliant upon health system capabilities and workforce availability. There is limited data available, as an issue of transparency regarding surveillance reporting, as well as 
health system capabilities that can provide information about regions and jurisdictions to support planning and policy making. This also includes missing information on community/regional infrastructure that provides information of the needs of those areas to support those people who require immediate treatment or isolation. Additionally, there is a need for information on satellite services, those health care (and other) services that are required to service communities, to regional and remote areas of Australia. This raises the issue of how to best identify those services with both the workforce capabilities including access to enough personal protective equipment to test people and reagent stocks for laboratories to keep up with testing demands. This is of particular concern for regional and remote areas, and areas servicing Aboriginal and Torres Strait Islander people.

There is also a need to further develop secure access to primary care electronic medical records (including ACCHO data) to provide information on existing care to the relevant service providers and to provide aggregated details to the relevant sectors to support the allocation of resources. This is of particular importance for those people who may require ongoing health care yet may not be accessing services during the pandemic. Primary health care data is also critical for identifying and improving vaccine coverage. Data development (in terms of accessibility) in primary health care should be a priority action area for Aboriginal and Torres Strait Islander people.

And lastly, there was an identified need for expansive health promotion efforts in language, particularly for those outside urban settings to prevent remote community spread. The ability of Land Councils and NAC$\mathrm{CHO}$ to support the mobilisation their 144 members enabled swift action regarding efforts to provide information to Aboriginal and Torres Strait Islander peoples and communities about COVID-19 [2]. Indeed, the first health promotion tools in language were developed through the Northern Land Council before The Plan or the 'National Aboriginal and Torres Strait Islander COVID-19 Management Plan' was enacted [18]. There is a need to support the development of the necessary infrastructure to securely reposit these education and health promotion tools into the future.

\subsection{Data quality}

The implementation of the Standard Indigenous Question across routinely collected data sets in Australia occurred in 1996. Despite this, there are still are well known gaps in the reporting of Aboriginal and Torres Strait islander people in Australia's routinely collected data and data used for official reporting purposes [15]. The following issues with data quality have been identified:

- Number of Aboriginal and Torres Strait Islander peoples tested

- Identification of Aboriginal and Torres Strait Islander cases

- Accuracy of Indigenous status

- Reporting requirements.

While COVID-19 is a notifiable disease, only those people tested are included in the cases and attack rates. For those positive cases, the public reporting of the completeness of Indigenous status occurred from March through to August with a mean of $89 \%$ completeness over the time period (Fig. 3). Completeness of Indigenous status is no longer being reported. The reason for the cessation of the public reporting of completeness rates have not been provided.

The accuracy of the identification of Aboriginal and Torres Strait Islander people with COVID-19 is typically reliant upon whether an individual (or a family member) is asked to self-identify in health services. Data collected on COVID-19 cases, that includes the Indigenous identifier, comes from pathology laboratories, hospitals, deaths data and through contact tracing.

It is optional for private businesses (such as private pathology laboratories) and private health services (including some providing primary health care) to have an Indigenous identifier on their collection forms as part of their data collections. This has long been an issue, which has impacted not only reporting on communicable diseases but also cancer, disease screening registries as well as Medicare Australia [19]. This impacts data quality and reporting consistency for official reporting purposes during a pandemic. Given the importance of testing information in the control of the pandemic, the absence of this vital information for Aboriginal and Torres Strait Islander people is a major concern.

Other data collections impacted by inaccurate Indigenous status and requiring further attention include deaths data, vaccine registries data and treatment data (including ambulance data, emergency department data and hospital admissions data). Additionally, there is also limited or no reporting on Indigenous status quality in contact tracing, which is of particular importance to reduce the spread and transmission of COVID-19 in Aboriginal and Torres Strait Islander communities.

Furthermore, the criteria for jurisdictional reporting under the NNDSS indicates that cases are reported 


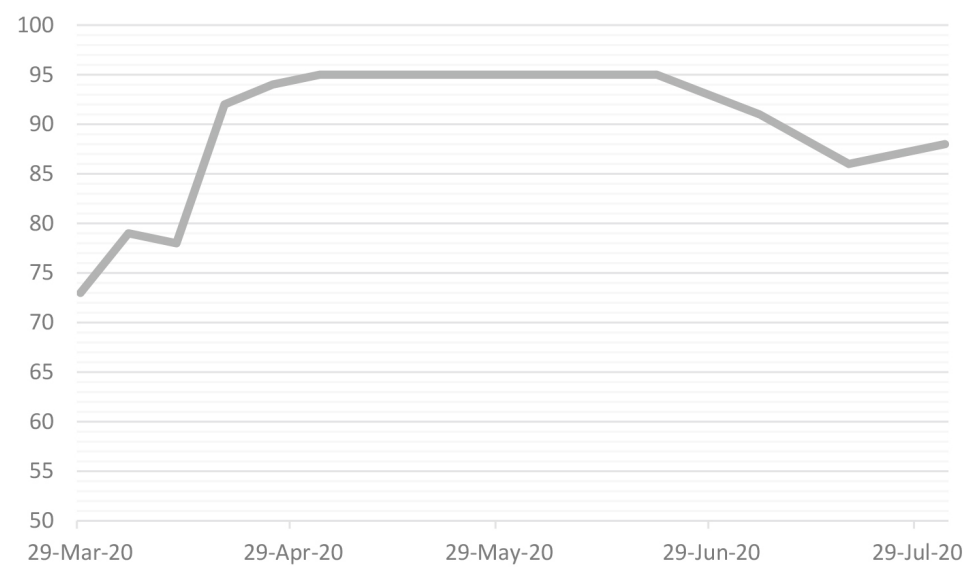

Fig. 3. Completeness (\%) of Indigenous status across Aboriginal and Torres Strait Islander COVID-19 cases publicly reported from March to August 2020. SOURCE: Commonwealth Department of Health, Communicable Diseases Intelligence COVID-19 Epidemiology Reports (all reports with Indigenous status completeness available included).

based on their Australian jurisdiction of residence rather than where they were detected. For example, a case reported previously in the NT for NSW resident is counted in the national figures as a NSW case [20]. This criteria, and resultant reporting, has the potential to impact public transparency in the disclosure of the risk of transmission, particularly at the beginning of the pandemic and highlights a potential risk that can be mitigated relatively easily.

\subsection{Provision of access to data relating to Aboriginal and Torres Strait Islander people}

There is limited involvement of Aboriginal and Torres Strait Islander people in the governance of the provision of access to data relating to Aboriginal and Torres Strait Islander people. There is a strong requirement to develop national governance processes that ensure the rights of Aboriginal and Torres Strait Islander people are met in regard to data collection and use within the nation. In August 2018, Prime Minister and Cabinet established the Office of the National Data Commissioner to build and support the infrastructure and use of public data [21]. Other national initiatives have included the National Collaborative Research Infrastructure Strategy and the developing National Health Information Strategy to make better use of research and health data [22,23]. There is limited description of Aboriginal and Torres Strait Islander governance within these national developments.

Two of the major issues with official data collections is the ease of which data can be shared and the interoperability of the data systems and platforms that are being used for the purposes of surveillance and report- ing. Australia is ahead of other nations in terms of data sharing and data linkage capabilities. However there is a requirement that Aboriginal and Torres Strait Islander people have mechanisms to govern their data and that these systems are harmonised in order to operationalise the data concerns raised in this article. Workforce planning also needs to take into consideration data to support public health preparedness and responsiveness. While Australia has a number of global public health experts and infectious disease leaders there is also a need to invest in the data infrastructure and capabilities of the nation in order to provide timely, accurate data in the preparedness, management and reporting of Aboriginal and Torres Strait Islander people.

\section{Concluding statement}

Aboriginal and Torres Strait Islander people have long asserted that they know what is best for themselves and their communities. The outstanding success during the COVID-19 pandemic has been the result of prompt and effective action by Aboriginal and Torres Strait Islander leaders and organisations and effective interactions with governments. The importance of effective processes in government and community that enables necessary, life-saving actions cannot be dismissed or underestimated. Additionally, the effectiveness of selfdetermination cannot be denied. The COVID-19 pandemic response is one of the strongest successful examples of what happens when Aboriginal and Torres Strait Islander people govern their own priorities and actions.

There are still many data issues regarding Aboriginal and Torres Strait Islander identification and reporting 
in pandemic surveillance. We have seen that the lack of identifier of pathology request forms results in no information for the crucial issue of testing rates. As we look to the future, data issues may also arise again with regards to vaccine coverage. A potential solution to this is to invest in population specific data governance within nations. For Aboriginal and Torres Strait Islander people, this is enacting self-determination in the collection and use of data. It is important to ensure that Aboriginal and Torres Strait Islander epidemiologists and demographers lead the way in discussions on data collection, quality and reporting during a pandemic and regarding official statistics. This is to enable existing data infrastructures and data systems to work for Aboriginal and Torres Strait Islander people and there are established mechanisms of expert voice as Aboriginal and Torres Strait Islander communities move closer towards data control and ownership within Australia.

\section{Acknowledgments}

The authors would like to acknowledge the work and other members of the International Group of Indigenous Health Measurement (IGIHM), particularly the Identification working group. They would also like to acknowledge the IGIHM co-chairs, Sam Notzon and Michele Connolly for their leadership and support. Authors would also like to acknowledge the funding and institutional support to undertake this work. Kalinda Griffiths was supported by the Scientia program with the Centre for Big Data Research in Health at UNSW.

\section{References}

[1] COVID-19 National Incident Room Surveillance Team. COVID-19 Australia: Epidemiology Report 31: Fortnightly reporting period ending 6 December 2020. 2020. Department of Health. Australian Government. doi: 10.33321/cdi.2020.44.92.

[2] Crooks, K., Casey, D., Ward, J.S. First Nations people leading the way in COVID-19 pandemic planning, response and management. The Medical Journal of Australia. 2020; Preprint - 29 April 2020. Accessed from https://www.mja.com.au/system/ files/2020-04/Crooks\%20preprint\%2029\%20April\%202020. pdf.

[3] Australian Bureau of Statistics. Estimates of Aboriginal and Torres Strait Islander Australians, June 2016. ABS cat. no. 3238.0.55.001. 2018. Canberra: ABS.

[4] Department of Health Australian Health Sector Emergency Response Plan for Novel Coronavirus (COVID-19) Management Plan for Aboriginal and Torres Strait Islander Populations. Australian Government. 2020. Accessed from https://www. health.gov.au/sites/default/files/documents/2020/03/australianhealth-sector-emergency-response-plan-for-novel-coronaviru s-covid-19-short-form-the-australian-health-sector-emergenc y-response-plan-for-novel-coronavirus-short-form.pdf.
[5] Department of Health. Australian Health Management Plan for Pandemic Influenza. Australian Government. ISBN: 978-174186-151-8. 2019. Accessed from https://www1.health.gov. au/internet/main/publishing.nsf/Content/519F9392797E2DDC CA257D47001B9948/\%24File/w-AHMPPI-2019.PDF.

[6] World Health Organisation. Weekly epidemiological update 8 December 2020. WHO. 2020. Accessed from https://www. who.int/publications/m/item/weekly-epidemiological-update 8-december-2020.

[7] Department of Health. Australian Health Sector Emergency Response Plan for Novel Coronavirus (COVID-19): Management plan for Aboriginal \& Torres Strait Islander communities, 2020. Australian Government. 2020. Accessed from https:// www.health.gov.au/resources/publications/management-plan -foraboriginal-and-torres-strait-islander-populations.

[8] Aboriginal and Torres Strait Islander Advisory Group on COVID-19. Communique Update: 15 October 2020. Australian Government. Date viewed <https://www.health.gov.au/ sites/default/files/documents/2020/10/aboriginal-and-torresstrait-islander-advisory-group-on-covid-19-communiques15-october-2020-communique_0.pdf>

[9] Dudgeon, P., Derry, K.L., Wright, M., et al. A National COVID-19 Pandemic Issues Paper on Mental Health and Wellbeing for Aboriginal and Torres Strait Islander Peoples. Transforming Indigenous Mental Health and Wellbeing Grant, The University of Western Australia Poche Centre for Indigenous Health. 2020. Accessed from https://apo.org.au/sites/default/ files/resource-files/2020-06/apo-nid306661.pdf.

[10] Naidu, L., Chiu, C., Habig, A., Lowbridge, C., Jayasinghe, S., Wang, H., McIntyre, P., Menzies, R. Vaccine preventable disease and vaccination coverage in Aboriginal and Torres Strait Islander people, Australia 2006-2010. Communicable Diseases Intelligence. 2013; 37(Supp). Australian Government Department of Health.

[11] Al-Yaman, F. The Australian Burden Of Disease Study: impact and causes of illness and death in Aboriginal and Torres Strait Islander people, 2011. Public Health Res Pract. 2017; 27(4): 2741732. doi: 10.17061/phrp2741732.

[12] Wolff, G.G. Influenza vaccination and respiratory virus interference among department of defense personnel during the 2017-2018 influenza season. Vaccine. 2020; 38(2): 350-354. doi: 10.1016/j.vaccine.2019.10.005.

[13] Kidd, M.R. Five principles for pandemic preparedness: lessons from the Australian COVID-19 primary care response. British Journal of General Practice. 2020; 70(696): 316-317. doi: 10.3399/bjgp20X710765.

[14] Flint, S.M., Davis, J.S., Su, J.-Y., Oliver-Landry, E.P., Rogers, B.A., Goldstein, A., Thomas, J.H., Parameswaran, U., Bigham, C., Freeman, K., Goldrick, P., Tong, S.Y.C. Disproportionate impact of pandemic (H1N1) 2009 influenza on Indigenous people in the Top End of Australia's Northern Territory. Medical Journal of Australia. 2010; 192(10): 617-622. doi: 10.5694/j.1326-5377.2010.tb03654.x.

[15] Griffiths, K., Coleman, C., Al-Yaman, F., Cunningham, J., Garvey, G., Whop, L., Jackson Pulver, L., Ring, I., Madden, $\mathrm{R}$. The identification of Aboriginal and Torres Strait Islander people in official statistics and other data: critical issues of international significance. Statistical Journal of the IAOS. 2019; 35(1): 91-106. doi: 10.3233/SJI-180491.

[16] Griffiths, K., Coleman, C., Lee, V., Madden, R. How colonisation determines social justice and Indigenous health - a review of the literature. J Pop Research. 2016; 33: 9-30. doi: 10.1007/s12546-016-9164-1.

[17] Langton, M., Mazel, O., Palmer, L., Shain, K., Tehan, M. 
(eds). Settling with Indigenous People: Modern treaty and agreement-making. The Federation Press. 2006. Sydney, NSW. ISBN: 978-1-86287-618-7.

[18] Keene, M. COVID-19 and Indigenous Australians: a chronology [1]. Research Papers, Parliament of Australia. 2020. Accessed from https://www.aph.gov.au/About_Parliament/Parlia mentary_Departments/Parliamentary_Library/pubs/rp/rp2021/ Chronologies/COVID19-IndigenousAustralians.

[19] Australian Institute of Health and Welfare. The inclusion of Indigenous status on pathology request forms. Cat. no. IHW 103. 2013. ISBN: 978-1-74249-493-7. Accessed from https://www.aihw.gov.au/reports/indigenous-australians/indi genous-status-on-pathology-request-forms/contents/table-ofcontents.

[20] Department of Health. COVID-19 current situation and case numbers Australian Government. Accessed from https://www. health.gov.au/news/health-alerts/novel-coronavirus-2019ncov-health-alert/coronavirus-covid-19-current-situationand-case-numbers.
[21] Department of Prime Minister and Cabinet. Best practice guide to applying data sharing principles. Australian Government. 2019. Accessed From https://www.pmc.gov.au/sites/default/ files/publications/data-sharing-principles-best-practice-guide -15-mar-2019.pdf.

[22] Australian Government. 2016 National Research Infrastructure Roadmap. 2017. Accessed from https://www.education.gov. au/2016-national-research-infrastructure-roadmap.

[23] National Health Information Independent Expert Panel. Accessed from https://www.aihw.gov.au/our-services/committe es/national-health-information-strategy-independent-e. 